\title{
Development of a Wireless Data Transmission System used for Power Transformer On-line Calibration
}

\author{
Lei Min, Xiong Qianzhu, Hu Haoliang, Li He, Li Dengyun, \\ Yang Chunyan
}

China Electric Power Research Institute, Wuhan, 430074, China

Keywords: transformer; wireless transmission; on-line calibration; ARM processor

\begin{abstract}
The design principle and structure of the wireless data transmission devices used for power transformer on-line calibration was described in this paper, design ideas and processes of the low-power hardware and software was introduced in detail. A prototype of the low-power and high anti-interference wireless data transmission device was developed, and a field test was conducted in the substation. The experiment indicates that the wireless data transmission device can transmit the experimental data steadily, and you can update the device program via wirelessly, reaching the purposes of transformer on-line calibration.
\end{abstract}

\section{Introduction}

High-Voltage Transformer, as an important component of gateway electrical energy metering device, is the authorized measuring instruments used for fair and just trade settlement 、 correct calculation and inner technic \& economic index for assessing power system between power generation companies and power grid corporations, power and supply companies, power supply companies and users. At present, offline calibration of the power transformer is mainly to meet periodic verification of transformers as electrical energy metering device. There is a huge number of power transformers in power system in China, it will be a huge amount of work and time consuming for testing the error performance during a power outage, and can not get the real data of three-phase line state ${ }^{[1]}$. Research of on-line calibration technology helps to overcome the above drawbacks of off-line calibration of the power transformer, and improves the level of uncertainty of error performance measurement of transformer, it lays a solid foundation for achieving the overall online calibration of electrical energy metering device and inproves the accuracy of electrical energy measurement ${ }^{[2,3]}$.

In order to achieve automatic real-time on-line calibration of measuring transformers in $110 \mathrm{kV} \sim 220 \mathrm{kV}$ voltage level gateway metering point, the conventional standard voltage and current transformers must be transformed into easy-install, light-weight and small-size transformer because of the factors of large size and weight which make it impossible to meet the needs of online calibration. But it will result in lower output power, smaller signal amplitude, easily influenced by external electromagnetic interference and it's not conducive to signal accuracy and long-distance transmission $^{[4,5]}$, so it must be converted into a digital signal with data acquisition unit and then transmitted. Insulation need to be taken into account when the digital signal is transmitted to the ground since the standard current transformer is placed on high-voltage lines, fiber-optic transmission is relatively good in data stability, but it has many defects such as that fiber insulator needs to be customized, high cost, and fiber is fragile and easily broken, which results in higher maintenance costs. To solve the problem, wireless data transmission devices is used in this paper to solve the insulation problems and simplify field wiring strength. It greatly reduces the chance of wiring errors and field wiring density by setting up the wireless LAN, and transferring experimental data from multiple devices to the data processing terminal wirelessly, thereby reducing the security risks of experimental site of substation and reaching the purpose of field on-line calibration. 


\section{Online Calibration System Block Diagram}

Fig. 1 shows a block diagram about wireless data transmission system of field on-line calibration system for transformer, the standard transformer on site includes a standard capacitive divider voltage transformer and a open standard current transformer, and their accuracy reached $0.05 \%$, each standard transformers are equipped with a wireless data transmission device which makes it possible for error correction of voltage and current transformer wirdlessly on site.

Since the real-time values of voltage and current signals need to be collected and packed to send in high speed in error calibration experiments, the sampling frequency of data acquisition unit is set to $4 \mathrm{kHz}$, and data for each sample point after calculating process occupies at least 3 bytes, so the bandwidth for data transmission of each transformer will be up to around $110 \mathrm{kbps}$ with other additional flag characters. Due to the large data volume and high real-time requirements of data transmission, it's necessary to configure the error correction section as part of a separate network segment and configure a separate reception device for data reception, the purpose of the above is to ensure real-time and reliability of data transmission. Device for measuring secondary voltage drop and load of voltage transformer is used to test the secondary circuit of transformer on site, each unit is equipped with a wireless data transmission device, the amount of data transmission of each unit is small and less than $10 \mathrm{kbps}$ and real-time requirements are not high, so another network segment for data reception is configured for measuring devices.

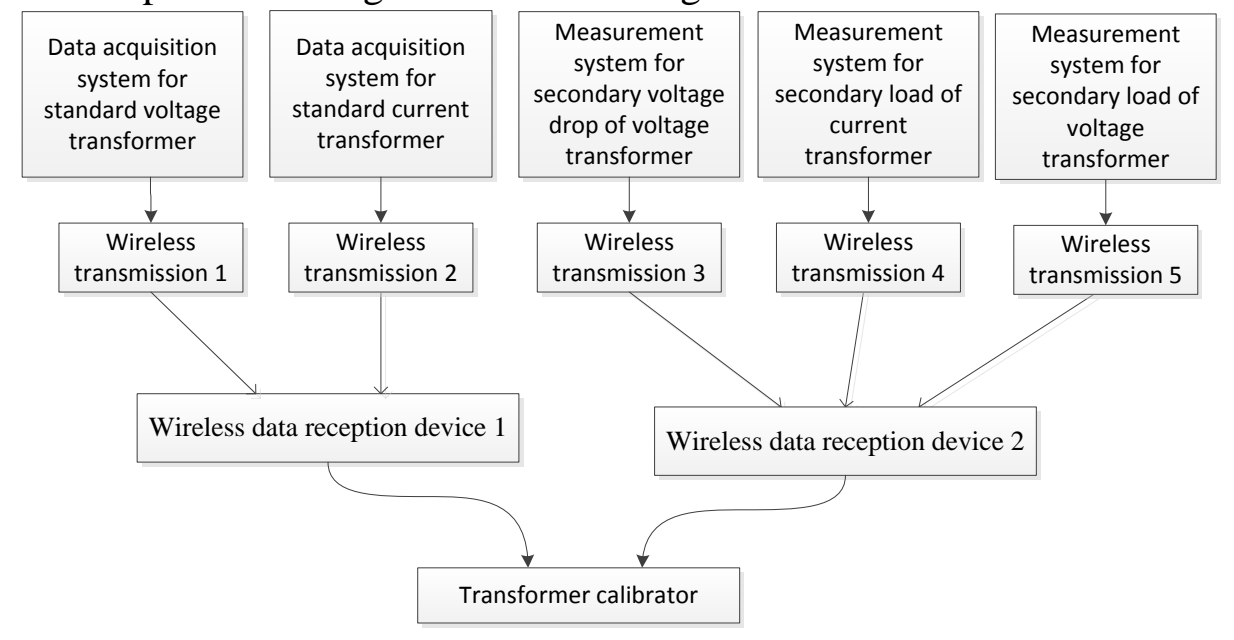

Fig.1. the structure of the wireless data transmission devices

\section{Design of Data Transmission Device}

\section{Development of Data Processing and Wireless Transmission Hardware Platform.}

After considering the power and speed of data processing apparatus, this device uses a 32-bit enhanced ARM processor STM32F103VET6 from STM32 family as the core of data processing platform for data caching, data packaging, operation and control. STM32 family is based on ARM Cortex-M3 core which is specially designed for embedded applications with high performance, low cost and low-power, clock frequencies of the enhanced series is up to $72 \mathrm{MHz}$, it not only has built-in flash memory whose density ranges between 32 and 128 Kbytes, reset circuit, low voltage detector, voltage regulators and precise $\mathrm{RC}$ oscillator, but also the high-performance on-chip resources, such as dual 12bit ADC, $4 \mathrm{Mbit} / \mathrm{sec}$ UART, $18 \mathrm{Mbit} / \mathrm{sec}$ SPI, full-speed USB 2.0 interface, it has the highest performance in similar products ${ }^{[6]}$. What is worth mentioning is that it supports on-line program upgrades via UART1, so it's convenient to modify error calibration value on site with wireless module used for upgrading program of processor, and it's helpful for future maintenance ${ }^{[7,8]}$.

There are many types of wireless data transmission module, since the environment of substations site is worse, and some substations have restrictions on the frequency of wireless products, so 
wireless data transmission module based on 2.4G common frequency is chosen. After several field tests for Bluetooth, NRF2401 module and Wi-Fi module, a WI-FI module named as WIFI232 with serial and Ethernet port is chosen as wireless data transmission module when taking stability, power and bandwidth and other factors into account ${ }^{[9,10]}$. The module has a standard RS232 serial interface and Ethernet port, you can choose one of them, data transmission mode can be transparent transmission and protocol transmission can also be used, the bandwidth can reach 4Mbit / s, fully able to meet the bandwidth claim of data transmission device. Because of the open operating instruction of WIFI232 module, parameters of the module can be configured by software on site, this function can be used to update program of STM32 processor. Fig.2 shows the block diagram of the circuit, Reset is a reset port of WIFI232 module and controlled by PE0 port, the configuration of the module can be modified in cooperation with the serial port and AT command operation; nReady and nLink are the status indication port used for indicating the completion of initiation and network connection respectively, you will know whether the data is transmitted efficiently while reading the status of PE1 and PE2 port. PA9 and PA10 are the data transmission and reception port of serial port UART1, so the program of STM32 processor can be updated wirelessly by WIFI232 module.

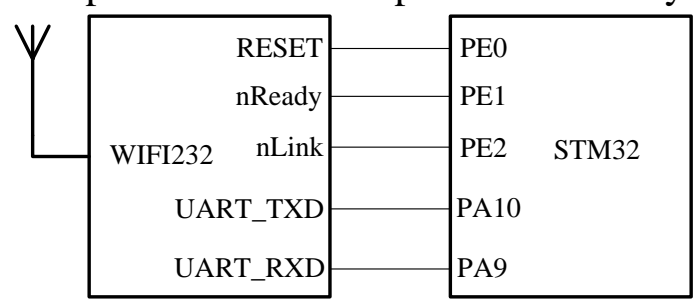

Fig.2. the connections of STM32 processor and WIFI232

\section{Software Design of Data Transmission Device}

Software flowchart of the data transmission device is shown in Fig.3.

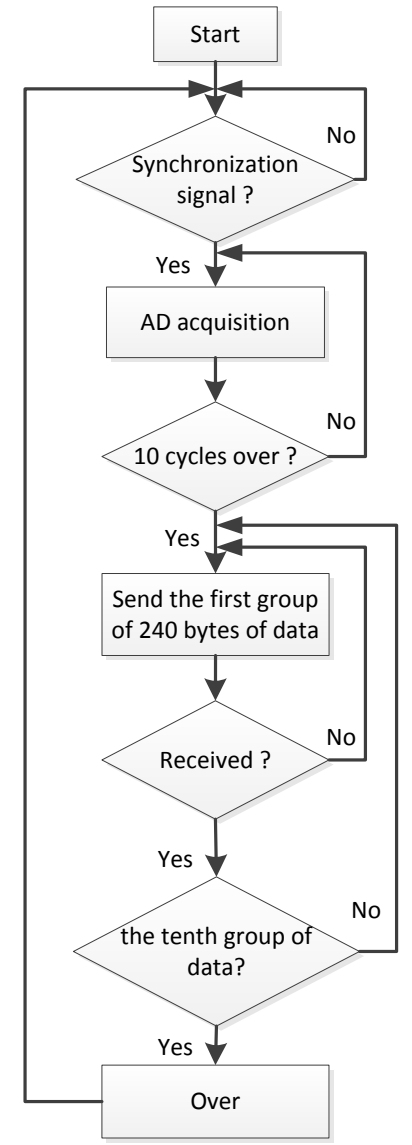

Fig.3. the software flow chart of the wireless data transmission device 
Serial communication is one of the most convenient transparent transmission modes, but there will be the risk of packet loss. Since the A/D sampling data is stored FIFO buffer by point in chronological order, sampling data is transmitted after 10 cycles via a wireless data transmission device, so as long as there is a loss of data at some point, the data behind will shift, resulting in FFT operation failure of data processing platform terminal, eventually leading to greater transformer error calculated values than normal value. In addition, the substation electromagnetic environment is bad, part of the wireless transmission device is disposed above the high-voltage lines, and electromagnetic interference is serious, in order to solve the above problems, protocol transmission mode is used in this system to ensure the reliability of data transmission by applying handshake protocol, CRC checksum and data retransmission mechanism.

A/D sample data includes a total of 2400 bytes in 10 cycles, which includes 80 sample points in each cycle and 3 bytes in each sample point, sample data of each cycle is packed and sent in transmitting terminal, reception terminal will check CRC, the receiving terminal will send confirmation command for allowing the transmission of data in next cycle when no error occurs, otherwise, it will send retransmission command, the transmitting terminal will retransmit data packet until it receives confirmation command. Fig.4 shows the data packet structure.

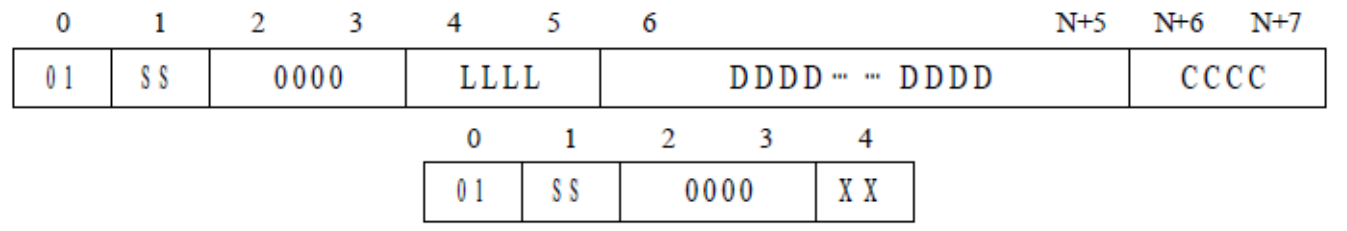

Fig.4. data packet structure and response command structure

Where the first row shows data packet structure, the first byte is command word, which indicates that data is sent from the collection terminal to the receiving terminal; the second byte is data packet sequence number, data packet sequence number and reply sequence number is one to one correspondence, ensuring that data transmission queue will be not displaced. The 3th and 4th byte is address byte, which is used to distinguish data sent by each data acquisition device. The 5th and 6th byte indicates the length of data packet. Bytes after the 7th byte are sample data and last two bytes is CRC checksum of the data packet. The second row shows data response command issued by the receiving terminal; the meaning of the first four bytes are the same as that of data packet structure, and the last byte is reply command, where 00 represents that data received is incorrect, non- 00 indicates incorrect and the data need to be retransmitted.

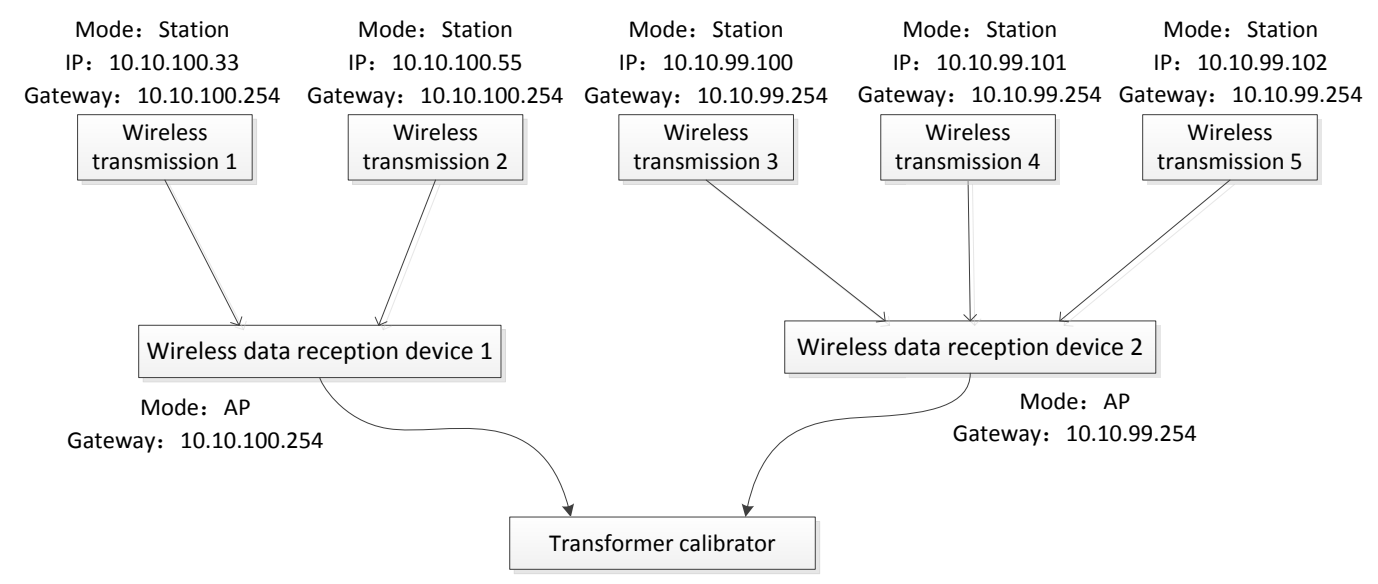

Fig.5. Mode of the wireless data transmission device and IP configuration

\section{Results and Analysis of System Operation}

The wireless data transmission module shows a good stability and fast data transmission speed after a long test, it meets system requirements. But there are some problems about the standard current transformer when high-voltage line is applied, the data cannot be transferred, it is found after several tests that the transmitting antenna of the device generates a corona. The antenna is 
$25 \mathrm{~cm}$ long and placed on top of standard current transformer, thus forming the tip discharge phenomenon, this problem is then solved by moving the antenna to the side of the transformer, and appropriately adding the equalizing ring.

After the final test on substation site, wireless data transmission system performs well and stable in data transmission work, the test results of transformers on-line verification system is shown in Fig.6.

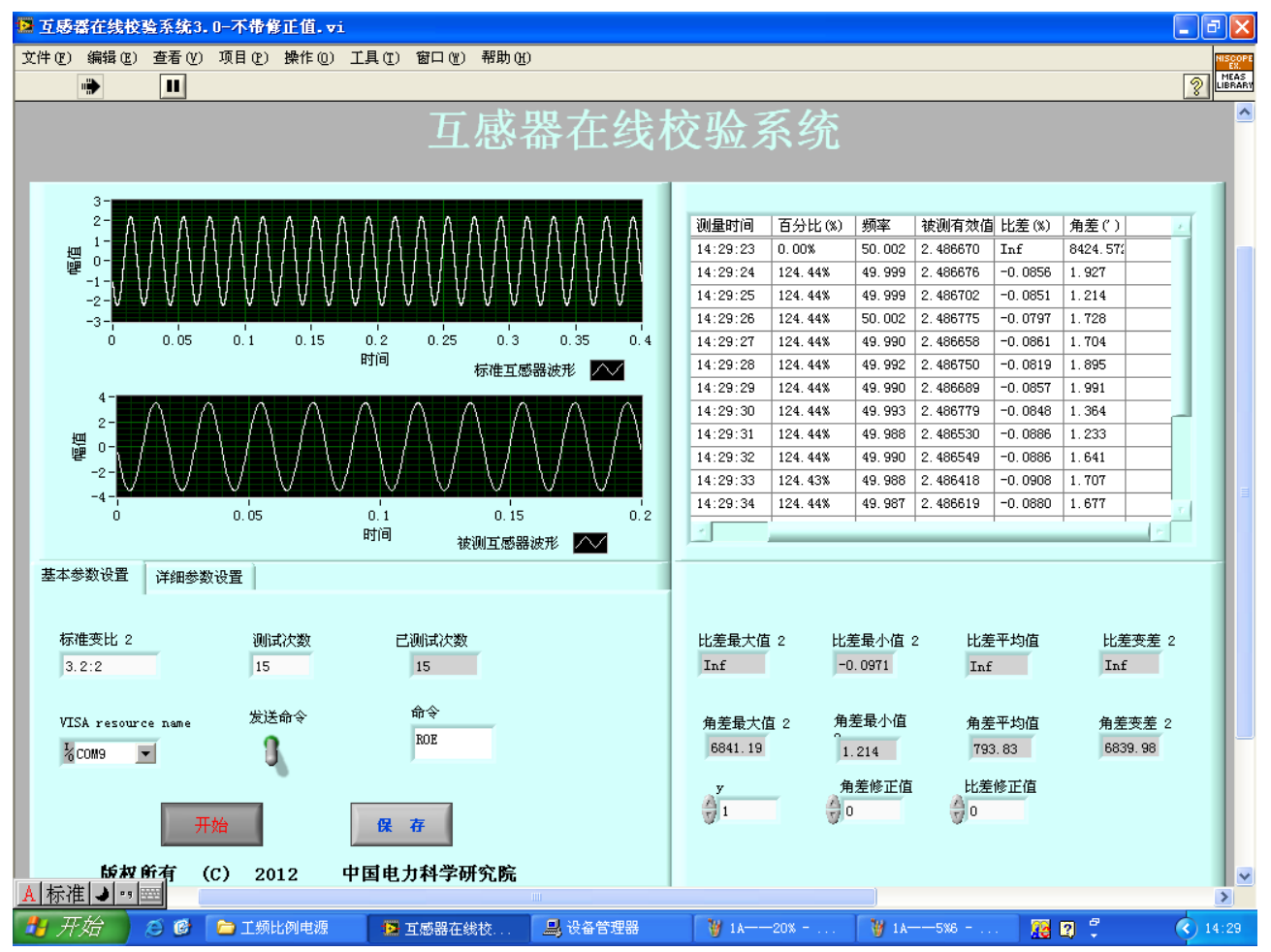

Fig.6. the software interface of transformers on-line calibration system

The waveform shown on the left and top side is standard current transformer waveform that transmitted by a wireless data transmission, and the processing results of transformer data is shown on the right side, the measurement results indicate that angle difference and ratio difference of the current transformer is stable and less volatile, indicating that the data transmitted by the wireless transmission device is not shifted or wrong on the top of the high-voltage lines, and wireless data transmission device has a good anti-jamming capability.

This paper describes the design principle and structure of wireless data transmission device used for power transformer on-line calibration, and details of design and processes of the device's hardware and software. The wireless data transmission device prototype developed with low-power, high anti-interference conducted a field test on substation, it simplifies field device wiring and increases the experimental security, and its stability achieves the desired purpose. The wireless data transmission device provides a new way for charged Verification of the power transformer, which has great value for application.

\section{References}

[1] Hu Haoliang, Li Qian, Lu Shufeng, et al. Comparison of Two Electronic Transformer Error Measuring Methods. High Voltage Engineering, 2011, 37(12):3022-3027.

[2] Zhao Zhanlei, Wang Jianguo. Analysis on the Relation between Error Size of Power Transformer and Electric Quantity. SCI-TECH Information development \& Economy. 2009, 35: 211-212.

[3] Yang Shihai, Xu Qing, Lu Shufeng. Field Testing and Analyzing of Instrument Transformer at the Power Exchange Point in Electric Power Grid to Electric Energy Balancing. Electrical Measurement \& Instrumentation. 2009, 9(46): 35-38. 
[4] Zhang Shuhan, Lei Min, Xiong Qianzhu. Development of Data Acquisition Device Used for Power Transformer on-line Calibration. Electrical measurement \& Instrumentation. 2012, 49(564): 82-85.

[5] Tan Hongen, Hu Haoliang, Lei Min, et al. Experimental Analysis of On-site Calibration of Electronic Instrument Transformer, High Voltage Engineering, 2010, 12(36): 2990-2995.

[6] W. Yonghong, X. Wei, H. Liping. ARM Cortex-M3 Microcontrollers Principles and Practice of STM32 Series, Beihang University Press, Beijing, 2008: 318-338.

[7] Zhang Xu, Qi Xueguang, Li Shiguang. Design of electric power data acquisition system based on STM32. Electronic Measurement Technology. 2010, 11(33): 90-93.

[8] Li Ping, Ling Li. Design and Implementation of Data Acquisition System Based on Embedded Nios II. Ship Electronic Engineering. 2012,1(211):74-76.

[9] Wang Chao, Luo Dehan,Zheng Wei, et al. Design of Embedded Intelligent Home Wireless Gateway Based on STM32. Computer technology and development2013, 23(3):241-244.

[10] Zeng Lei, Zhang Haifeng, Hou Weiyan. Design and Implement of WiFi Wireless Measurement and Control Network. Electrical measurement \& Instrumentation. 2011, 48(547): 81-84. 